Alessio Campisi ${ }^{1 *}$, Andrea Dell'Amore ${ }^{1 *}$, Luca Bertolaccini', Costantino Ricci' ${ }^{2}$, Alessandra Cancellieri' ${ }^{2}$, Franco Stella

'Department of Cardiothoracic Surgery, S. Orsola Malpighi Hospital, Bologna, Italy

${ }^{2}$ Department of Pathology, S. Orsola Malpighi University Hospital, Bologna, Italy

*The Authors equally contributed to the paper

\title{
Sialadenoma papilliferum of the bronchus: a rare tumour of salivary gland origin
}

\begin{abstract}
Sialadenoma papilliferum is a benign salivary tumour which rarely occurs in the bronchial tree. Up to now, only four cases of pulmonary papillary sialadenoma have been reported in the literature.

We discuss the case of a male patient with an accidental finding of a middle lobe nodule. The patient underwent a minimally invasive anatomical resection of the lobe to remove the lesion; the postoperative course was regular, and he was healthy at the last follow-up.
\end{abstract}

Key words: sialadenoma papilliferum, rare lung cancer, VATS, lobectomy

Adv Respir Med. 2020; 88: 267-270

\section{Introduction}

Sialadenoma papilliferum (SP) is a rare, benign, papillary salivary gland tumour, usually diagnosed as a white exophytic mass of the oral and maxillofacial region [1]. Since its first description in 1969 [2], less than 100 cases have been reported in the medical literature, and most of them are located in the palate.

This paper aims to report the fifth case, to the best of our knowledge, of an SP involving the bronchial tree.

\section{Case description}

A 66-year-old male was referred to our Department for an incidental finding of a solitary pulmonary nodule of the middle lobe. The patient's medical history included renal transplantation two years earlier from a living donor due to renal failure from mesangial IgA deposits. Eight months after the transplantation, the man underwent right nephrectomy of the native kidney due to a clear cell renal carcinoma in stage pT1a
No M0. During the standard oncological follow-up, a whole-body computed tomography (CT) scan showed a $12 \times 13 \mathrm{~mm}$ solitary pulmonary nodule of the middle lobe (Figure 1) with fluorodeoxyglucose (18F-FDG) avidity (SUVmax 4.3) at the positron emission tomography (PET) (Figure 2). A transbronchial biopsy was not feasible, and the CT-guided transthoracic biopsy was not diagnostic. Pulmonary function tests were regular. Therefore, we discussed the indication for surgery during the multidisciplinary oncological team meeting. A VATS middle lobectomy with systematic lymphadenectomy through a biportal approach was performed. The postoperative period was uneventful, and the patient was discharged on the third postoperative day. After almost three years from surgery, he is healthy, with no recurrence.

Pathologically, at the gross examination of the resected lobe, a micro-cystic neoplasm of $1.5 \mathrm{~cm}$ in diameter, was found close to the lobar bronchus.

Frozen sections showed a papillary and cystic lesion with no sign of malignancy and the diagnosis was deferred.

Address for correspondence: Alessio Campisi, Department of Cardiothoracic Surgery, S. Orsola Malpighi Hospital, Bologna, Italy; e-mail: alessio.campisi@studio.unibo.it DOI: 10.5603/ARM.2020.0111

Received: 09.11.2019

Copyright (C) 2020 PTChP

ISSN 2451-4934 

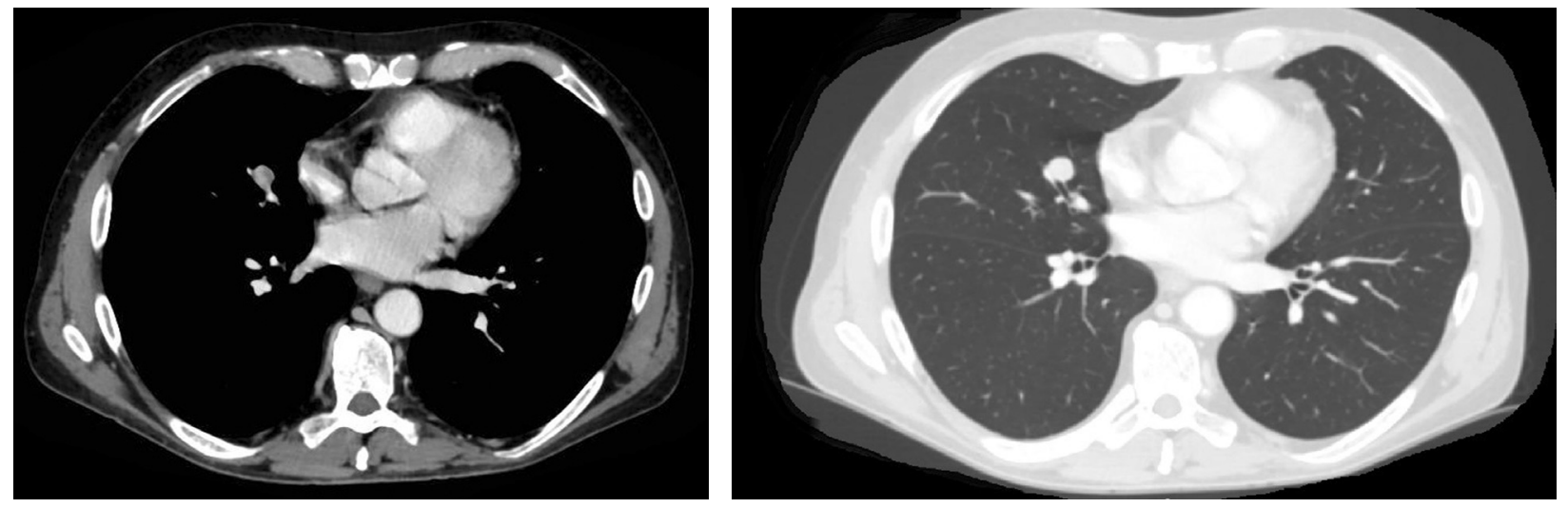

Figure 1. CT scan showing the middle lobe nodule
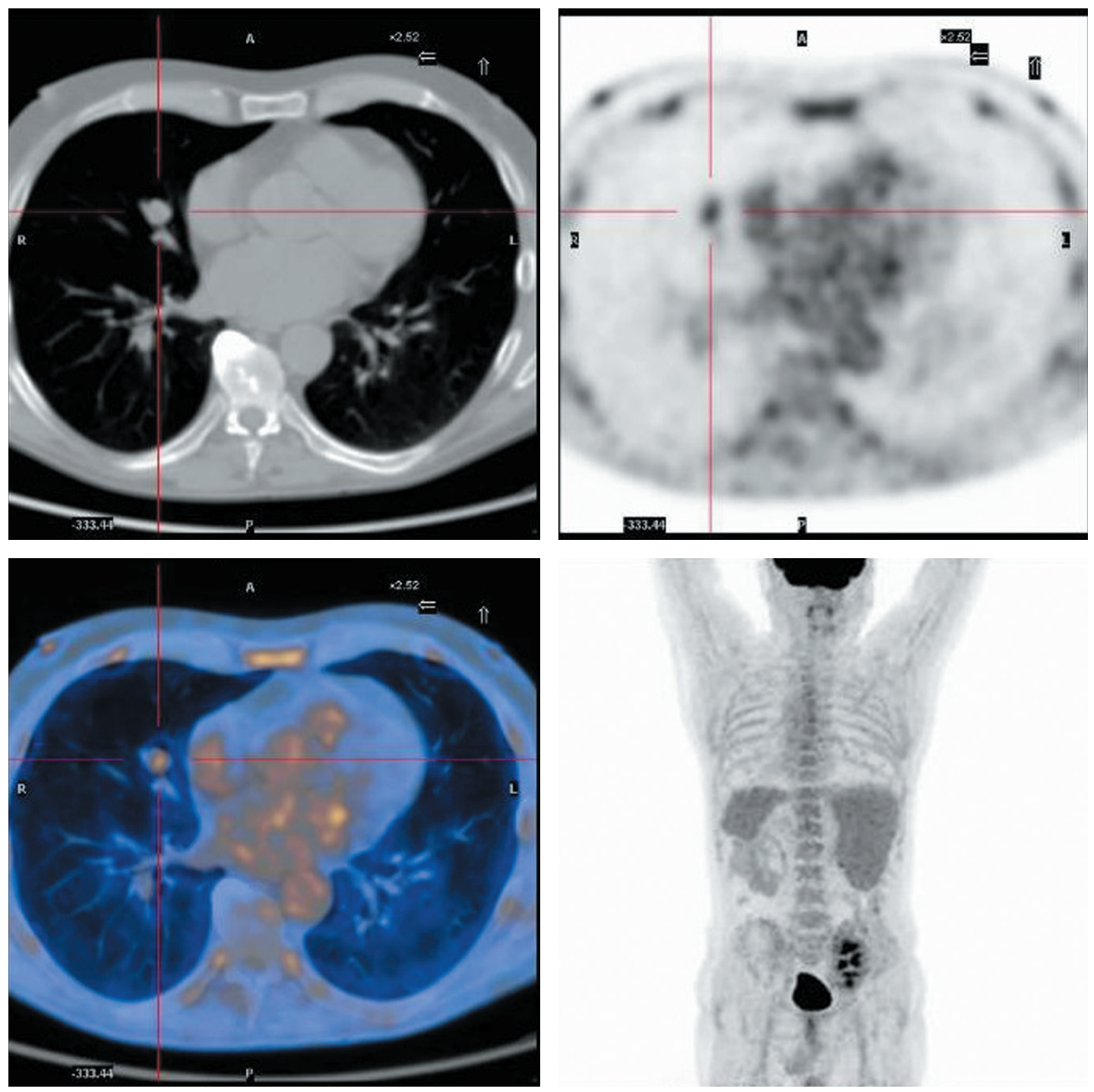

Figure 2. PET 18 F-FDG showing the contrast uptake of the nodule 

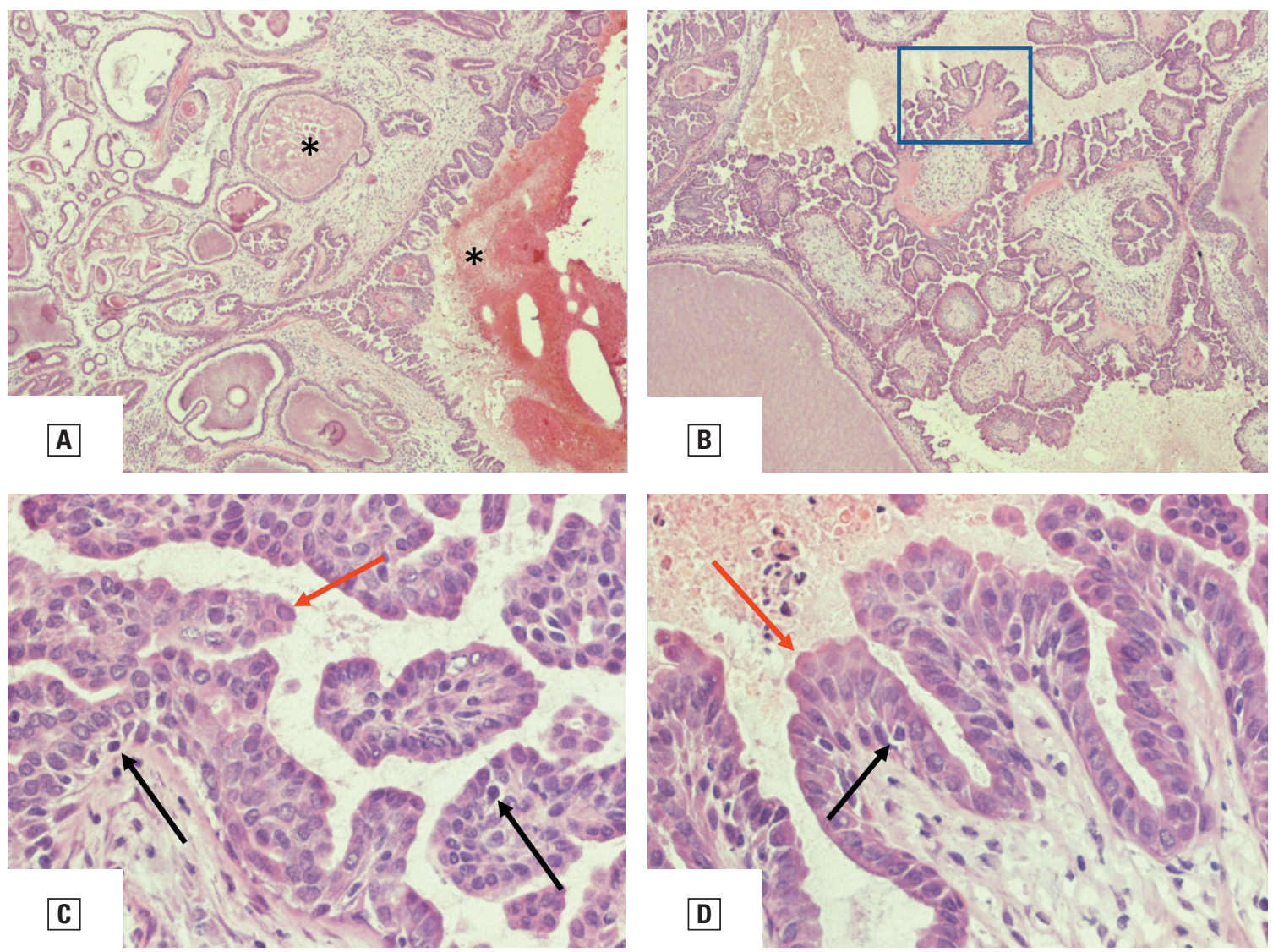

Figure 3. A., B. At low power, both cysts with blood and eosinophilic material (asterisks) and cysts with papillary projections are observed (blue square) (H\&E, original magnification $100 \times$ ); C., D. Papillary projections and cysts are lined by a luminal epithelium (luminal cells, red arrows) showing pluristratification, anisonucleosis and nucleoli and a basal monostratified epithelium (basal or aluminal cells, black arrows) with a myoepithelial appearance (H\&E, original magnification $400 \times$ )

Histologically, the lesion was described as a cystic neoplasm, well-demarcated by a thin fibrous capsule, with cysts containing eosinophilic material or papillary structures lined by at least two layers of the epithelium (Figure 3). At immunohistochemistry, neoplastic cells were negative for TTF1, Napsin A, Thyroglobulin, PAX8, and GATA3. The epithelial cells of the basal layer were positive at immunohistochemistry with p63 and ck14. The suprabasal layer was negative for p63 and only focally positive for ck14; it was often multi-layered, with some micropapillary tufts, and showed mild polymorphic hyperchromatic nuclei and rare prominent nucleoli. Furthermore, the cysts containing eosinophilic material were lined by the same layered epithelium that was negative for TTF1, Napsin A, Thyroglobulin, PAX8, and GATA3 as well. The lesion seems to originate from submucosal bronchial glands.

The described findings are consistent with a cystic papillary neoplasm of undetermined malignant potential (probably, low), most likely originating from bronchial glands. The final dia- gnosis was sialadenoma papilliferum (SP), a rare subset of ductal papilloma of minor salivary gland origin, also described in the bronchi [3-5]. In our opinion, this tumour could be considered as a "salivary gland type bronchial neoplasm".

\section{Conclusion}

Rare primary tumours of the lung account for less than $1 \%$ of all primary lung neoplasms and include a wide variety of cancers [6]. They usually present as a non-small cell lung cancer with symptoms related to their size, localisation, and malignancy (cough, dyspnoea, chest pain, haemoptysis, fever, fatigue, etc.). A tissue biopsy is often mandatory to make an accurate diagnosis, and surgery is still the most effective treatment, with chemotherapy and radiotherapy playing a role as adjuvant strategies.

Here, we present the fifth case of SP of the tracheobronchial tree published in the literature. A thorough search was performed in PubMed on December $1^{\text {st }}, 2018$, using the terms "lung", "bron- 
chial" and "sialadenoma/sialadenoma papilliferum" as keywords. Only four studies were found (one was written in German and was excluded). First described in 1969 by Abrams and Finck [2], SP is a benign, neoplasm of salivary gland origin which is named after its histological resemblance to syringocystadenoma papilliferum of cutaneous adnexal origin [7]. In the oral cavity, it appears as a white-coloured, exophytic, papillary lesion of the mucosa $[1,7]$. In the three reports analysed, the macroscopic appearance is similar, being described as a well-demarcated, white, papillary or verrucous, sessile to a pedunculated lesion of the trachea or the bronchus [3-5]. It shows a histopathological biphasic growth with an exophytic papillary squamous component and a solid/cystic part. SP is slightly more common among males, with a male-to-female ratio of 1.6:1, mainly affecting individuals $>50$ years of age (mean standard \pm deviation $56.8 \pm 15.5$ years) [1]. SP has been reported in a variety of locations characterised by slow growth and a benign course, although few cases of recurrence have been documented [8]. A malignant transformation is still unique, although possible [7].

Besides, the cell of origin of SP is still controversial, but this neoplasm is thought to arise from both the excretory duct cells and bronchial glands in the case of salivary gland tumours and tracheobronchial tree lesions, respectively [3]. According to the literature review [8], the recommended treatment is a conservative surgical approach. In the three reported pulmonary cases, two anatomical resections were performed (a sleeve right upper lobectomy and a lower right lobectomy); in the third one, simple excision of the endotracheal mass was made. In all cases, patients have been free of disease for at least 6 months after surgery.

In our case, we did not have a preoperative diagnosis: therefore, considering the mass as a malignant tumour and evaluating its localisation, we performed a middle lobectomy. Our patient underwent a periodical follow-up CT scan due to the significant risk of recurrence of kidney malignancy under immunosuppression, and he is currently free of tumour.

In conclusion, we report the fifth case of SP occurring in the bronchus. The awareness of the existence of such rare lesions is essential for pathologists, and clinicians should keep in mind the possible occurrence of this intrabronchial exophytic tumoral lesion in the differential diagnosis. It is also mandatory to consider the chance, although improbable, of malignancy for these lesions. Undoubtedly, additional reports with longer follow-up are needed to demonstrate the most appropriate treatment of this very rare lesion.

\section{Conflict of interest}

None declared.

\section{References:}

1. Kubota Y, Kubo C, Mori Y. Sialadenoma papilliferum of the hard palate: a case report. J Oral Maxillofac Surg. 2012; 70(7): 1609-1612, doi: 10.1016/j.joms.2011.08.012, indexed in Pubmed: 22079070.

2. Abrams AM, Finck FM. Sialadenoma papilliferum. A previously unreported salivary gland tumor. Cancer. 1969; 24(5): 1057-1063, doi: 10.1002/1097-0142(196911)24:5<1057::aid -cncr2820240529>3.0.co;2-1, indexed in Pubmed: 5353939.

3. Honda Y, Shiraishi K, Nomori H, et al. Sialadenoma papilliferum of the bronchus: rare tracheobronchial tumor of salivary gland type. Pathol Int. 2009; 59(8): 572-576, doi: 10.1111/i. 1440-1827.2009.02408.x, indexed in Pubmed: 19627541.

4. Grzesik P, Passik C, Mahfoozi A. An unusual cause of dyspnea. Ann Thorac Surg. 2016; 102(4): e333-e334, doi: 10.1016/j. athoracsur.2016.02.051, indexed in Pubmed: 27645975.

5. Bobos M, Hytiroglou P, Karkavelas G, et al. Sialadenoma papilliferum of bronchus. Virchows Arch. 2003; 443(5): 695699, doi: 10.1007/s00428-003-0879-y, indexed in Pubmed: 12937978.

6. Block MS, Vierkant RA, Rambau PF, et al. Australian Ovarian Cancer Study Group. Rare pulmonary neoplasms. Mayo Clin Proc. 1993; 68(5): 492-498, doi: 10.1016/s0025-6196(12)601992, indexed in Pubmed: 8386792.

7. Shimoda M, Kameyama K, Morinaga S, et al. Malignant transformation of sialadenoma papilliferum of the palate: a case report. Virchows Arch. 2004; 445(6): 641-646, doi: 10.1007/ s00428-004-1091-4, indexed in Pubmed: 15455228.

8. Fowler CB, Damm DD. Sialadenoma papilliferum: analysis of seven new cases and review of the literature. Head Neck Pathol. 2018; 12(2): 193-201, doi: 10.1007/s12105-017-0852-8, indexed in Pubmed: 28887760. 